\title{
On the Representation Theorems of Poisson, Riemann and Volterra
} for the Euler-Poisson-Darboux Equation

\author{
F. G. FriedLander \& A. E. Heins
}

Communicated by A. ERDÉLYI

\section{Introduction}

This paper continues our study of the representation of solutions of the Euler-Poisson-Darboux equation [4]. This linear partial differential equation, of the hyperbolic type in two independent variables, has the form

$$
\frac{\partial^{2} \phi}{\partial t^{2}}=\frac{\partial^{2} \phi}{\partial r^{2}}+\frac{2 \alpha}{r} \frac{\partial \phi}{\partial r}
$$

where we have assumed $\alpha$ to be a real parameter such that $0<\alpha<1$. For a discussion of other real values of $\alpha$ we refer to [4] and [9]. This equation has been the object of much study, in part due to its interest in many specific problems in classical physics [3] and in part due to its intrinsic mathematical interest [9]. On the latter points, it is perhaps the most simple linear partial differential equation with a singular line $(r=0)$. As we have remarked in [4], the presence of this line poses certain questions which are beyond the scope of the standard theory.

There are three different representations known for equation (1.1), the first one having been given by Porsson [6] in 1823. It has the form

$$
\phi(t, r)=\int_{0}^{\pi} f(t+r \cos \psi) \sin ^{2 \alpha-1} \psi d \psi+r^{1-2 \alpha} \int_{0}^{\pi} g(t+r \cos \psi) \sin ^{1-2 \alpha} \psi d \psi
$$

for $0<\alpha<1, \alpha \neq 1 / 2$. For $0<\alpha<1 / 2$, the first integral dominates in the limit $r \rightarrow 0^{+}$, while for $1 / 2<\alpha<1$, the second one does. Given that $f(\tau)$ and $g(\tau) \in C^{2}$ in the interval $t-r \leqq \tau \leqq t+r$, we can show that (1.2) satisfies (1.1) and that

and

$$
\operatorname{Lim}_{r \rightarrow 0^{+}} \phi(t, r)=\frac{\Gamma(1 / 2) \Gamma(\alpha)}{2 \Gamma(\alpha+1 / 2)} f(t)
$$

$$
\operatorname{Lim}_{r \rightarrow 0^{+}} r^{2 \alpha} \frac{\partial \phi}{\partial r}=\frac{\Gamma(1 / 2) \Gamma(1-\alpha)}{\Gamma(1 / 2-\alpha)} g(t)
$$

provided that $0<\alpha<1 / 2$. If $1 / 2<\alpha<1$, these limits can be read as finite parts in the sense of Hadamard. The two integrals in (1.2) are clearly linearly independent save for the case $\alpha=1 / 2$. 
For the exceptional case $\alpha=1 / 2$ we write (1.2) in the form (following DARBoux [2])

$$
\begin{aligned}
\phi(t, r)= & \int_{0}^{\pi}\left[\frac{f(t+r \cos \psi)}{2}-\frac{g(t+r \cos \psi)}{1-2 \alpha}\right] \sin ^{2 \alpha-1} \psi d \psi \\
& +r^{1-2 \alpha} \int_{0}^{\pi}\left[\frac{f(t+r \cos \psi)}{2}+\frac{g(t+r \cos \psi)}{1-2 \alpha}\right] \sin ^{1-2 \alpha} \psi d \psi .
\end{aligned}
$$

In the limit $\alpha \rightarrow 1 / 2,(1.2 \mathrm{a})$ becomes

$$
\phi(t, r)=\int_{0}^{\pi} f(t+r \cos \psi) d \psi+\int_{0}^{\pi} g(t+r \cos \psi) \ln \left(r \sin ^{2} \psi\right) d \psi
$$

so that in this case we have

$$
\operatorname{Lim}_{r \rightarrow 0^{+}} \phi(t, r)=\pi f(t) \text { and } \operatorname{Lim}_{r \rightarrow 0^{+}} r \frac{\partial \phi}{\partial r}=\pi g(t) .
$$

A second representation is to be found for the special case $\alpha=1 / 2$ in VOLTERRA's work [8] of 1892. For $0<\alpha<1$, equation (1.1) is satisfied by either of the following representations:

$$
\int_{0}^{\infty}\left[f_{1}(t+r \cosh \psi)+f_{2}(t-r \cosh \psi)\right] \sinh ^{2 \alpha-1} \psi d \psi
$$

or

$$
r^{1-2 \alpha} \int_{0}^{\infty}\left[f_{3}(t+r \cosh \psi)+f_{4}(t-r \cosh \psi)\right] \sinh ^{1-2 \alpha} \psi d \psi
$$

In order that (1.3) and (1.3a) be solutions of equation (1.1) we require order conditions on $f_{1}(\tau), f_{3}(\tau), f_{2}(-\tau)$ and $f_{3}(-\tau)$ as $\tau \rightarrow \infty$ as well as some smoothness conditions on these functions, and we shall discuss them in Section 3. Observe that (1.3) and (1.3a) employ data which are outside of the interval $(t-r, t+r)$. We have already discussed in [4], Section 8 , the interpretation of $f_{3}(t)+f_{4}(-t)$. Incidentally, LAMB [5] felt that for the special case $\alpha=1 / 2,(1.3)$ was preferable to (1.2) since (1.3) embraced both converging and diverging waves while (1.2) did not discriminate them. We shall see that this point is illusory.

The third representation was given by RIEMANN in 1860 in terms of what is now called a RIEMANN function [7]. In fact this one may be used to obtain $\phi(t, r)$ for $0<t<r$ when the data $\phi(t, r)$ and $\frac{\partial \phi}{\partial t}$ are assigned at $t=0$. If, however, such data are given when $0<t<r$, then there are some modifications in the results of RIEMANN, as COPSON [1] had pointed out in 1958. Copson had made extensive use of certain integral transforms to find these modifications. The results of POISSON, VOLTERRA and COPSON are all equivalent in the restricted interval of $\alpha$ which we employ. It is, of course, possible to extend this interval by methods which are standard for equation (1.1) [9], but we shall not pursue this point here. It is to be noted that we do not require data beyond the point $r+t$ on the $t$ axis. If $0<r<t$, assumptions regarding the behavior of $\phi$ and $\frac{\partial \phi}{\partial t}$ at $t=0$ in the neigh-
borhood of $r=0^{+}$will be needed. 
In order to demonstrate the equivalence of (1.2) and (1.3) we ask if it is possible to express them in terms of Cauchy data on the regular line $t=0, r>0$. Upon doing so, we encounter some simple Abel integral equations. These equations may be solved and upon simplification we are led directly to the Riemann representation for both of the cases $0<t<r$ and $0<r<t$. In the process we find Copson's extension of RIEMANN's result. We shall see that the PoIsson representation is the easiest one to handle.

\section{The Cauchy Problem for (1.2)}

Since the two integrals in (1.2) are linearly independent when $0<\alpha<1, \alpha \neq 1 / 2$, we shall consider two separate Cauchy problems when $\alpha \neq 1 / 2$. The exceptional case $\alpha=1 / 2$ will be discussed separately. We first examine the Cauchy problem on the line $t=0$ for the regular representation

$$
\phi_{1}(t, r)=\int_{0}^{\pi} f(t+r \cos \psi) \sin ^{2 \alpha-1} \psi d \psi
$$

That is, we shall show that we can rewrite (2.1) in terms of $\phi_{1}(r, t)=a_{1}(r)$ and $\frac{\partial \phi_{1}}{\partial t}=b_{1}(r)$, when $r \geqq 0$ and $t=0$, for $0<\alpha<1, \alpha \neq 1 / 2$. Then we shall show that the same may be done for the singular part

$$
\phi_{2}(t, r)=r^{1-2 \alpha} \int_{0}^{\pi} g(t+r \cos \psi) \sin ^{1-2 \alpha} \psi d \psi
$$

in terms of $\phi_{2}(t, r)=a_{2}(r)$ and $\frac{\partial \phi_{2}}{\partial t}=b_{2}(r)$, when $r \geqq 0$ and $t=0$, again for $0<\alpha<1, \alpha \neq 1 / 2$. The details of these problems are essentially the same and we shall only examine the particulars of the first one. In order to simplify the subsequent discussion, we shall decompose $\phi_{1}(t, r)$ and $\phi_{2}(t, r)$ into two parts, one of which vanishes while the other of which has a vanishing $t$ derivative at $t=0$. That is, we shall investigate the forms of the functions $\phi_{1}(t, r)$ and $\phi_{2}(t, r)$ which are respectively odd and even about the line $t=0$. We shall only discuss the cases which deal with the vanishing of $\phi_{1}$ and $\phi_{2}$ since the other two cases are essentially the same. Now (2.1) may be rewritten with the substitution $\tau=r \cos \psi$ as

$$
\phi_{1}(t, r)=\int_{0}^{r} \sin ^{2 \alpha-2} \psi[f(t+\tau)+f(t-\tau)] d \tau=\int_{0}^{r} \frac{[f(t+\tau)+f(t-\tau)] d \tau}{r^{2 \alpha-1}\left[r^{2}-\tau^{2}\right]^{1-\alpha}}
$$

From the condition $\phi_{1}(0, r)=0$, we conclude that $f(\tau)+f(-\tau)=0$. When the derivative condition is employed we obtain

$$
b_{1}(r)=\int_{0}^{r} \frac{\left[f^{\prime}(\tau)-f^{\prime}(-\tau)\right] d \tau}{r^{2-1}\left[r^{2}-\tau^{2}\right]^{1-\alpha}} .
$$

where the prime indicates differentiation with respect to $\tau$. This integral may be inverted to give

$$
[f(\lambda)-f(-\lambda)]=2 \frac{\sin \pi \alpha}{\pi} \int_{0}^{\lambda} \frac{b_{1}(\tau) \tau^{2 \alpha} d \tau}{\left(\lambda^{2}-\tau^{2}\right)^{\alpha}}, \quad 0<\alpha<1, \quad \alpha \neq 1 / 2,
$$


since $b_{1}(\tau)$ is bounded and continuous when $t-r<\tau<t+r$. Since $f$ is odd, we find that

$$
f(\lambda)=-f(-\lambda)=\frac{\sin \pi \alpha}{\pi} \int_{0}^{\lambda} \frac{b_{1}(\tau) \tau^{2 \alpha} d \tau}{\left(\lambda^{2}-\tau^{2}\right)^{\alpha}}, \quad \lambda>0 .
$$

Now we have two cases to consider, depending on whether $r \gtrless t$. Let us suppose that $r<t$. Then

$$
\begin{aligned}
\phi_{1}(t, r)=\frac{\sin \pi \alpha}{\pi r^{2 \alpha-1}} & \left\{\int_{0}^{r}\left(r^{2}-\tau^{2}\right)^{\alpha-1} d \tau \int_{0}^{t+\tau} \frac{\lambda^{2 \alpha} b_{1}(\lambda) d \lambda}{\left[(t+\tau)^{2}-\lambda^{2}\right]^{\alpha}}\right. \\
& \left.+\int_{0}^{r}\left(r^{2}-\tau^{2}\right)^{\alpha-1} d \tau \int_{0}^{t-\tau} \frac{\lambda^{2 \alpha} b_{1}(\lambda) d \lambda}{\left[(t-\tau)^{2}-\lambda^{2}\right]^{\alpha}}\right\} .
\end{aligned}
$$

These integrals may be written in a more useable form by interchanging the order of integration and performing some rearrangements. We have then

$$
\begin{aligned}
\phi_{1}(t, r)=\frac{\sin \pi \alpha}{\pi r^{2 \alpha-1}} & \left\{\int_{0}^{t} \lambda^{2 \alpha} b_{1}(\lambda) d \lambda \int_{0}^{r}\left[r^{2}-\tau^{2}\right]^{\alpha-1}\left[(t+\tau)^{2}-\lambda^{2}\right]^{-\alpha} d \tau\right. \\
& +\int_{t}^{t+r} \lambda^{2 \alpha} b_{1}(\lambda) d \lambda \int_{\lambda-t}^{r}\left(r^{2}-\tau^{2}\right)^{\alpha-1}\left[(t+\tau)^{2}-\lambda^{2}\right]^{-\alpha} d \tau \\
& +\int_{0}^{t-r} \lambda^{2 \alpha} b_{1}(\lambda) d \lambda \int_{0}^{r}\left(r^{2}-\tau^{2}\right)^{\alpha-1}\left[(t-\tau)^{2}-\lambda^{2}\right]^{-\alpha} d \tau \\
& \left.+\int_{t-r}^{t} \lambda^{2 \alpha} b_{1}(\lambda) d \lambda \int_{0}^{t-\lambda}\left(r^{2}-\tau^{2}\right)^{\alpha-1}\left[(t-\tau)^{2}-\lambda^{2}\right]^{-\alpha} d \tau\right\} .
\end{aligned}
$$

If we now replace $\tau$ by $-\tau$ in the third and fourth integrals, $\phi_{1}(t, r)$ will simplify to

$$
\begin{aligned}
\phi_{1}(t, r)=\frac{\sin \pi \alpha}{\pi r^{2 \alpha-1}} & \left\{\int_{0}^{t-r} \lambda^{2 \alpha} b_{1}(\lambda) d \lambda \int_{-r}^{r}\left(r^{2}-\tau^{2}\right)^{\alpha-1}\left[(t+\tau)^{2}-\lambda^{2}\right]^{-\alpha} d \tau\right. \\
& \left.+\int_{t-r}^{t+r} \lambda^{2 \alpha} b_{1}(\lambda) d \lambda \int_{\lambda}^{r}\left(r^{2}-\tau^{2}\right)^{\alpha-1}\left[(t+\tau)^{2}-\lambda^{2}\right]^{-\alpha} d \tau\right\} .
\end{aligned}
$$

Several remarks should be made about equation (2.7). The first is that the inner integral in each term is an Eulerian integral which can be reduced to a more familiar form. In order to effect this, we apply a bilinear transformation to the $\tau$ integral with limits which run from $-r$ to $r$ such that the points $\tau(-\lambda-t$, $\lambda-t,-r, r)$ go into $\sigma(Z, \infty, 0,1)$ where

$$
Z=\frac{t^{2}-(r-\lambda)^{2}}{4 r \lambda}
$$

Then

$$
\int_{-r}^{r}\left(r^{2}-\tau^{2}\right)^{\alpha-1}\left[(t+\tau)^{2}-\lambda^{2}\right]^{-\alpha} d \tau=\frac{Z^{-\alpha} \lambda^{-\alpha} r^{\alpha-1} \Gamma^{2}(\alpha)}{2 \Gamma(2 \alpha)} F(\alpha, \alpha, 2 \alpha, 1 / Z)
$$

where $F(\alpha, \alpha, 2 \alpha, 1 / Z)$ is the hypergeometric function. Similarly if we apply a bilinear transformation to the $\tau$ integral which runs from $\lambda-t$ to $r$ such that 
$(\tau-r,-\lambda-t, r, \lambda-t)$ goes into $\sigma(\infty, Z, 1,0)$ we get

$$
\int_{\lambda-t}^{r}\left(r^{2}-\tau^{2}\right)^{\alpha-1}\left[(t+\tau)^{2}-\lambda^{2}\right]^{-\alpha} d \tau=\frac{\lambda^{-\alpha}}{2 r^{1-\alpha}} \frac{\pi}{\sin \pi \alpha} F(\alpha, 1-\alpha, 1, Z) .
$$

Hence we may rewrite equation (2.7) as

$$
\begin{gathered}
\phi_{1}(t, r)=\frac{\sin \pi \alpha}{2 \pi} \int_{0}^{t-r}\left(\frac{\lambda}{r Z}\right)^{\alpha} \frac{\Gamma^{2}(\alpha)}{\Gamma(2 \alpha)} F(\alpha, \alpha, 2 \alpha, 1 / Z) b_{1}(\lambda) d \lambda \\
+\frac{1}{2} \int_{t-r}^{t+r}\left(\frac{\lambda}{r}\right)^{\alpha} F(\alpha, 1-\alpha, 1, Z) b_{1}(\lambda) d \lambda
\end{gathered}
$$

which is valid for $r<t, 0<\alpha<1$.

The second remark deals with the observation that there are two different integrals required on the $\lambda$ axis for the case $r<t$. We therefore have a very specific continuation of $F(\alpha, 1-\alpha, 1, Z), t-r<\lambda<t+r$ into the interval $0<\lambda<t-r$. Indeed, if one draws characteristics from the point $(t, r)$ to the $t$ axis, there are intersections at the points $(t-r, 0)$ and $(t+r, 0)$. One of these characteristics will also intersect the $r$-axis at $(0, t+r)$ while the other characteristic drawn from $(t-r, 0)$ will intersect the $r$-axis at $(0, t-r)$. Accordingly, this second characteristic is the reflection of the one drawn through the point $(t, r)$ from the line $r=0$ and it is this one which brings about the two integrals in equation (2.7). The second integral is the conventional Riemann representation while the first one is a modification noted by CoPson [1] in 1958. The conventional Cauchy existence theorem does not apply here since one of the characteristics through the point $(t, r)$ intersects the singular line $r=0$ as well as the line $t=0$. Finally, since $f(\lambda) \in C^{2}$, $t-r \leqq \lambda \leqq t+r$, the PoISSON representation for the regular case (2.1) implies that $\phi_{t}=f^{\prime}(t)$ at $r=0$ and $\phi_{t r}=0, r \rightarrow 0^{+}$. From the first conclusion we have that $b_{1}\left(0^{+}\right)=f^{\prime}(0)$ and from the second one we have that $d b_{1} / d r=0, r \rightarrow 0^{+}$. Since $\phi_{t r}$ exists at $t=0$, it clearly satisfies a Lipschitz condition in $r$.

We now turn to the case $r>t$. Here we have

$$
\begin{aligned}
\phi_{1}(t, r)=\frac{\sin \pi \alpha}{\pi r^{2 \alpha-1}} & \left\{\int_{0}^{t} \lambda^{2 \alpha} b_{1}(\lambda) d \lambda \int_{0}^{r}\left(r^{2}-\tau^{2}\right)^{\alpha-1}\left[(t+\tau)^{2}-\lambda^{2}\right]^{-\alpha} d \tau\right. \\
& +\int_{t}^{t+r} \lambda^{2 \alpha} b_{1}(\lambda) d \lambda \int_{\lambda-t}^{r}\left(r^{2}-\tau^{2}\right)^{\alpha-1}\left[(t+\tau)^{2}-\lambda^{2}\right]^{-\alpha} d \tau \\
& +\int_{0}^{t} \lambda^{2 \alpha} b_{1}(\lambda) d \lambda \int_{0}^{t-\lambda}\left(r^{2}-\tau^{2}\right)^{\alpha-1}\left[(t-\tau)^{2}-\lambda^{2}\right]^{-\alpha} d \tau \\
& \left.-\int_{0}^{r-t} \lambda^{2 \alpha} b_{1}(\lambda) d \lambda \int_{t+\lambda}^{r}\left(r^{2}-\tau^{2}\right)^{\alpha-1}\left[(t-\tau)^{2}-\lambda^{2}\right]^{-\alpha} d \tau\right\} .
\end{aligned}
$$

The form of the last integral arises from the requirement that it is necessary to employ (2.5) when the argument of $f$ is negative. Upon replacing $\tau$ by $-\tau$ in the third integral and noting that

$$
\int_{\lambda=t}^{r}\left(r^{2}-\tau^{2}\right)^{\alpha-1}\left[(t+\tau)^{2}-\lambda^{2}\right]^{-\alpha} d \tau=\int_{\lambda+t}^{r}\left(r^{2}-\tau^{2}\right)^{\alpha-1}\left[(t-\tau)^{2}-\lambda^{2}\right]^{-\alpha} d \tau,
$$


we get

(2.8a) $\phi_{1}(t, r)=\frac{\sin \pi \alpha}{\pi r^{2 \alpha-1}} \int_{r-t}^{r+t} \lambda^{2 \alpha} b_{1}(\lambda) d \lambda \int_{\lambda-t}^{r}\left(r^{2}-\tau^{2}\right)^{\alpha-1}\left[(t+\tau)^{2}-\lambda^{2}\right]^{-\alpha} d \tau$.

The relation (2.9) may be derived by applying the bilinear transformation

$$
\tau=\frac{2 t r^{2}+\left(t^{2}-\lambda^{2}+r^{2}\right) \sigma}{t^{2}-\lambda^{2}+r^{2}+2 t \sigma}
$$

to the integral on the right side of the equality. Upon employing the same bilinear transformation which we did in (2.7), we may reduce (2.8a) to

$$
\phi_{1}(t, r)=\frac{1}{2} \int_{r-t}^{r+t}\left(\frac{\lambda}{r}\right)^{\alpha} F(\alpha, 1-\alpha, 1, Z) b_{1}(\lambda) d \lambda, \quad r>t, \quad 0<\alpha<1 .
$$

The Cauchy problem for $\phi_{2}(t, r)$ on the line $t=0$ (when $\phi_{2}(0, r)=0$ ) follows without any major changes in method. Indeed for $r<t$

$$
\begin{aligned}
\phi_{2}(t, r)= & \frac{\sin \pi \alpha}{\pi}\left\{\int_{0}^{t-r} \lambda b_{2}(\lambda) d \lambda \int_{-r}^{r}\left(r^{2}-\tau^{2}\right)^{-\alpha}\left[(t+\tau)^{2}-\lambda^{2}\right]^{\alpha-1} d \tau\right. \\
& \left.\quad+\int_{t-r}^{t+r} \lambda b_{2}(\lambda) d \lambda \int_{\lambda-t}^{r}\left(r^{2}-\tau^{2}\right)^{-\alpha}\left[(t+\tau)^{2}-\lambda^{2}\right]^{\alpha-1} d \tau\right\} \\
= & \frac{\sin \pi \alpha}{2 \pi} \frac{\Gamma^{2}(1-\alpha)}{\Gamma(2-2 \alpha)} \int_{0}^{t-r} Z^{\alpha-1}\left(\frac{\lambda}{r}\right)^{\alpha} b_{2}(\lambda) F(1-\alpha, 1-\alpha, 2-2 \alpha, 1 / Z) d \lambda \\
& +\frac{1}{2} \int_{t-r}^{t+r}\left(\frac{\lambda}{r}\right)^{\alpha} b_{2}(\lambda) F(1-\alpha, \alpha, 1, Z) d \lambda .
\end{aligned}
$$

For $r>t$, we get

$$
\begin{aligned}
\phi_{2}(t, r) & =\frac{\sin \pi \alpha}{\pi} \int_{r-t}^{r+t} \lambda b_{2}(\lambda) d \lambda \int_{\lambda-t}^{r}\left(r^{2}-\tau^{2}\right)^{-\alpha}\left[(t+\tau)^{2}-\lambda^{2}\right]^{\alpha-1} d \tau \\
& =\frac{1}{2} \int_{r-t}^{r+t}\left(\frac{\lambda}{r}\right)^{\alpha} b_{2}(\lambda) F(1-\alpha, \alpha, 1, Z) d \lambda .
\end{aligned}
$$

Both (2.10) and (2.10a) are valid for $0<\alpha<1$. The kernels in (2.8b) and (2.10a) are the same due to the fact that $F(\alpha, 1-\alpha, 1, Z)=F(1-\alpha, \alpha, 1, Z)$, but there are some distinct differences in the kernels of (2.7a) and (2.10). Note that since $g(\lambda) \in C^{2}$ in an interval which includes the origin, we have $\frac{\partial}{\partial r}\left(r^{2 \alpha-1} b_{2}\right)=0, r \rightarrow 0^{+}$ and $r^{2 \alpha} \frac{\partial \phi_{2}}{\partial r}=\sqrt{\pi} \frac{\Gamma(1-\alpha)}{\Gamma\left(\frac{1}{2}-\alpha\right)} g(t), r \rightarrow 0^{+}$when $0<\alpha<1, \alpha \neq 1 / 2$.

There is no difficulty in treating the exceptional case $\alpha=1 / 2$. If we use (1.2a) to determine $\phi_{1}(t, r)$ and $\phi_{2}(t, r)$, we shall acquire the situation which we discussed in Section 1 of [4] in terms of $b_{1}(r)$ and $b_{2}(r)$.

\section{The Volterra Representation for (1.1)}

We have seen in Section 2 that the PoIsson representation for equation (1.1) could be converted into a Riemann representation for $0<r<t$ or $0<t<r$, when $0<\alpha<1$. This representation makes use of data between $t-r$ and $t+r$ on the 
$t$-axis. As such it uses data on the singular line $r=0$ which has been cut out by the characteristics of (1.1) drawn from the point $(t, r)$. In 1892, VolTERRA gave another set of representations for equation (1.1) in the special case $\alpha=1 / 2$. The data they require are now to be found in the complement of the interval of length $2 r$ which is symmetric about a point on the $t$-axis. For $0<\alpha<1$, his representations assume the forms

$$
\begin{gathered}
\int_{0}^{\infty} f_{1}(t+r \cosh \psi) \sinh ^{2 \alpha-1} \psi d \psi, \\
\int_{0}^{\infty} f_{2}(t-r \cosh \psi) \sinh ^{2 \alpha-1} \psi d \psi, \\
r^{1-2 \alpha} \int_{0}^{\infty} f_{3}(t+r \cosh \psi) \sinh ^{1-2 \alpha} \psi d \psi,
\end{gathered}
$$

and

$$
r^{1-2 \alpha} \int_{0}^{\infty} f_{3}(t-r \cosh \psi) \sinh ^{1-2 \alpha} \psi d \psi .
$$

We can show subject to appropriate behavior of $f_{i}(\lambda), i=1,3$ and $f_{i}(-\lambda), i=2,4$ when $\lambda \rightarrow \infty$, as well as some differentiability properties of $f_{i}( \pm \lambda)$, that (3.1) and (3.2) satisfy (1.1) ([4], Section 8).

There are, however, truncated versions of (3.1) and (3.2) which are more useful for our purposes. If for example, $f_{1}(\lambda)=f_{2}(-\lambda)=0$, for $\lambda>L>0$, we can write

$$
\Phi_{1}(t, r)=\int_{0}^{\psi_{0}} f_{1}(t+r \cosh \psi) \sinh ^{2 \alpha-1} \psi d \psi
$$

and

$$
\Phi_{2}(t, r)=\int_{0}^{\psi} f_{2}(t-r \cosh \psi) \sinh ^{2 \alpha-1} \psi d \psi
$$

where

$$
\psi_{0}(t, r, L)=\ln \frac{L-t+\sqrt{(L-t)^{2}-r^{2}}}{r}
$$

and

$$
\psi_{1}(t, r, L)=\ln \frac{L+t+\sqrt{(L+t)^{2}-r^{2}}}{r}
$$

and $-L<t<L$. Under the hypotheses that $f_{1}(\lambda)$ and $f_{2}(-\lambda) \in C^{2},|\lambda|<L$ and $0<\alpha<1$, a direct calculation will show that $\Phi_{1}(t, r)$ and $\Phi_{2}(t, r)$ are solutions of (1.1). This being the case, if we now form a linear combination of $\Phi_{1}(t, r)$ and $\Phi_{2}(t, r)$ and ask whether this combination implies a regular Cauchy problem on the line $t=0, r>0$, we shall see that the results of Section 2 are rederived.

We therefore investigate the same Cauchy problem which we did in Section 2 using $\Phi_{1}(t, r)$ and $\Phi_{2}(t, r)$ as a solution of equation (1.1) and the initial conditions $\Phi_{1}(0, r)+\Phi_{2}(0, r)=0$ and $\partial\left[\Phi_{1}(t, r)+\Phi_{2}(t, r)\right] / \partial t=b_{1}(r), r>0, t=0$. From the first initial condition we get immediately

$$
f_{1}(\tau)+f_{2}(-\tau)=0 \quad r<\tau<L
$$


From the second one, we get an Abel-type integral equation, that is,

$$
b_{1}(r)=\frac{f_{2}(-L)-f_{1}(L)}{\left(L^{2}-r^{2}\right)^{1-\alpha}}+\int_{r}^{L} \frac{\left[\frac{\partial f_{1}}{\partial \tau}-\frac{\partial f_{2}}{\partial \tau}\right] d \tau}{r^{2 \alpha-1}\left(\tau^{2}-r^{2}\right)^{1-\alpha}}, \quad r>0
$$

where now the functions $f_{1}$ and $f_{2}$ under the integral sign depend only on $\tau$. This integral equation may be solved, and we get

$$
f_{1}(r)-f_{2}(-r)=-\frac{2 \sin \pi \alpha}{\pi} \int_{r}^{L} \frac{\tau^{2 \alpha} b_{1}(\tau) d \tau}{\left(\tau^{2}-r^{2}\right)^{\alpha}} \text {. }
$$

From (3.3) and (3.4) we may therefore conclude that

$$
f_{1}(r)=-f_{2}(-r)=-\frac{\sin \pi \alpha}{\pi} \int_{r}^{L} \frac{\tau^{2 \alpha} b_{1}(\tau) d \tau}{\left(\tau^{2}-r^{2}\right)^{\alpha}}, \quad r>0 .
$$

Let us observe that we need a condition such as $b_{1}(\tau) \in C^{\prime}, 0 \leqq \tau \leqq L$ in order to write (3.4).

In order to show that $\Phi_{1}(t, r)+\Phi_{2}(t, r)$ can be written as a representation of the Riemann type, we start with the aid of (3.1 c) and (3.1 d) and put

$$
\Phi_{1}(t, r)+\Phi_{2}(t, r)=\int_{r}^{L-t} \frac{f_{1}(t+\tau) d \tau}{r^{2 \alpha-1}\left(\tau^{2}-r^{2}\right)^{1-\alpha}}+\int_{r}^{L+t} \frac{f_{2}(t-\tau) d \tau}{r^{2 \alpha-1}\left(\tau^{2}-r^{2}\right)^{1-\alpha}} .
$$

At this point it becomes clear that we should distinguish the cases $r>t$ and $r<t$. For $r>t, f_{2}(t-\tau)=-f_{1}(\tau-t)$ and therefore (3.5) becomes

$$
\begin{aligned}
\Phi_{1}(t, r)+\Phi_{2}(t, r)=-\frac{\sin \pi \alpha}{\pi} & \left\{\int_{r}^{L-t} \frac{d \tau}{\left(\tau^{2}-r^{2}\right)^{1-\alpha}} \int_{t+\tau}^{L} \frac{\lambda^{2 \alpha} b_{1}(\lambda) d \lambda}{\left[\lambda^{2}-(t+\tau)^{2}\right]^{\alpha}}\right. \\
& \left.-\int_{r}^{L+t} \frac{d \tau}{\left(\tau^{2}-r^{2}\right)^{1-\alpha}} \int_{\tau-t}^{L} \frac{\lambda^{2 \alpha} b_{1}(\lambda) d \lambda}{\left[\lambda^{2}-(\tau-t)^{2}\right]^{\alpha}}\right\} .
\end{aligned}
$$

We can now show that for $L>t,(3.6)$ is independent of $L$. This is accomplished by calculating the partial derivative of the right hand side of (3.6) with respect to $L$ and showing that it is identically zero. Indeed, this partial derivative is

$$
-\frac{\sin \pi \alpha}{\pi}\left\{\int_{r}^{L-t} \frac{L^{2 \alpha} b_{1}(L) d \tau}{\left[\tau^{2}-r^{2}\right]^{1-\alpha}\left[L^{2}-(t+\tau)^{2}\right]^{\alpha}}-\int_{r}^{L+\tau} \frac{L^{2 \alpha} b_{1}(L) d \tau}{\left[\tau^{2}-r^{2}\right]^{1-\alpha}\left[L^{2}-(\tau-t)^{2}\right]^{\alpha}}\right\} .
$$

On the other hand

$$
\int_{r}^{L+t} \frac{d \tau}{\left[\tau^{2}-r^{2}\right]^{1-\alpha}\left[L^{2}-\left(t+\tau^{2}\right)\right]^{\alpha}}=\int_{r}^{L-t} \frac{d \tau}{\left[\tau^{2}-r^{2}\right]^{1-\alpha}\left[L^{2}-(\tau-t)^{2}\right]^{\alpha}}
$$

which we can show as we did in Section 2. Since (3.6) is independent of $L$, it leads directly to a Volterra type of representation (the case $L \rightarrow \infty$ ) or we may put $L=t+r$ in which case we get

$$
\Phi_{1}(t, r)+\Phi_{2}(t, r)=\frac{\sin \pi \alpha}{\pi} \int_{r}^{r+2 t} \frac{d \tau}{\left[\tau^{2}-r^{2}\right]^{\alpha}} \int_{\tau-t}^{t+r} \frac{\lambda^{2 \alpha} b_{1}(\lambda) d \lambda}{\left[\lambda^{2}-(\tau-t)^{2}\right]^{1-\alpha}} .
$$


Upon interchanging the order of integration (3.7) becomes

$$
\Phi_{1}(t, r)+\Phi_{2}(t, r)=\frac{\sin \pi \alpha}{\pi} \int_{r-t}^{r+t} \lambda^{2 \alpha} b_{1}(\lambda) d \lambda \int_{r}^{\lambda+t} \frac{d \tau}{\left[\tau^{2}-r^{2}\right]^{\alpha}\left[\lambda^{2}-(\tau-t)^{2}\right]^{1-\alpha}}
$$

However, with the aid of an appropriate bilinear transformation we may write

$$
\int_{r}^{\lambda+t} \frac{d \tau}{\left[\tau^{2}-r^{2}\right]^{\alpha}\left[\lambda^{2}-(\tau-t)^{2}\right]^{1-\alpha}}=\int_{-r}^{t-\lambda} \frac{d \tau}{\left[\tau^{2}-r^{2}\right]^{\alpha}\left[(\tau-t)^{2}-\lambda^{2}\right]^{1-\alpha}}
$$

and upon replacing $\tau$ by $-\tau$ in the last integral this becomes

$$
\int_{\lambda-t}^{r} \frac{d \tau}{\left(\tau^{2}-r^{2}\right)^{\alpha}\left[(\tau+t)^{2}-\lambda^{2}\right]^{1-\alpha}}
$$

Hence (3.8) is precisely (2.8a).

Now we turn to the case $0<r<t$. Here we have from (3.5) that

$$
\begin{aligned}
\Phi_{1}(t, r)+\Phi_{2}(t, r) \\
=\int_{r}^{L-t} \frac{f_{1}(t+\tau) d \tau}{r^{2 \alpha-1}\left(\tau^{2}-r^{2}\right)^{1-\alpha}}+\int_{r}^{L+t} \frac{f_{2}(t-\tau) d \tau}{r^{2 \alpha-1}\left(\tau^{2}-r^{2}\right)^{1-\alpha}} \\
=\int_{r}^{L-t} \frac{f_{1}(t+\tau) d \tau}{r^{2 \alpha-1}\left(\tau^{2}-r^{2}\right)^{1-\alpha}}+\int_{r}^{t} \frac{f_{2}(t-\tau) d \tau}{r^{2 \alpha-1}\left(\tau^{2}-r^{2}\right)^{1-\alpha}}-\int_{t}^{L+t} \frac{f_{1}(\tau-t) d \tau}{r^{2 \alpha-1}\left(\tau^{2}-r^{2}\right)^{1-\alpha}} \\
=\frac{\sin \pi \alpha}{r^{2 \alpha-1} \pi}\left\{-\int_{r}^{L-t} \frac{d \tau}{\left(\tau^{2}-r^{2}\right)^{1-\alpha}} \int_{\tau+t}^{L} \frac{\lambda^{2 \alpha} b_{1}(\lambda) d \lambda}{\left[\lambda^{2}-(t+\tau)^{2}\right]^{\alpha}}\right. \\
\left.\quad+\int_{r}^{t} \frac{d \tau}{\left(\tau^{2}-r^{2}\right)^{1-\alpha}} \int_{t-\tau}^{L} \frac{\lambda^{2 \alpha} b_{1}(\lambda) d \lambda}{\left[\lambda^{2}-(t-\tau)^{2}\right]^{\alpha}}+\int_{t}^{L+t} \frac{d \tau}{\left(\tau^{2}-r^{2}\right)^{1-\alpha}} \int_{\tau-t}^{L} \frac{\lambda^{2 \alpha} b_{1}(\lambda) d \lambda}{\left[\lambda^{2}-(\tau-t)^{2}\right]^{\alpha}}\right\} .
\end{aligned}
$$

But (3.9) is independent of $L$ so that if we put $L=t+r$, we get for the case $0<r<t$

$$
\begin{aligned}
\Phi_{1}(t, r)+\Phi_{2}(t, r)= & \frac{\sin \pi \alpha}{\pi r^{2 \alpha-1}}\left\{\int_{r}^{t} \frac{d \tau}{\left[\tau^{2}-r^{2}\right]^{1-\alpha}} \int_{t-\tau}^{r+t} \frac{\lambda^{2 \alpha} b_{1}(\lambda) d \lambda}{\left[\lambda^{2}-(t-\tau)^{2}\right]^{\alpha}}\right. \\
& \left.+\int_{t}^{r+2 t} \frac{d \tau}{\left[\tau^{2}-r^{2}\right]^{1-\alpha}} \int_{\tau-t}^{r+t} \frac{\lambda^{2 \alpha} b_{1}(\lambda) d \lambda}{\left[\lambda^{2}-(\tau-t)^{2}\right]^{\alpha}}\right\} .
\end{aligned}
$$

If we now interchange the order of integration in these integrals and simplify the result of this, we get

$$
\begin{aligned}
\Phi_{1}(t, r)+\Phi_{2}(t, r)=\frac{\sin \pi \alpha}{\pi r^{2 \alpha-1}} & \left\{\int_{0}^{t-r} \lambda^{2 \alpha} b_{1}(\lambda) d \lambda \int_{t-\lambda}^{t+\lambda} \frac{d \tau}{\left[\tau^{2}-r^{2}\right]^{\alpha}\left[\lambda^{2}-(t-\tau)^{2}\right]^{1-\alpha}}\right. \\
& \left.+\int_{t-r}^{t+r} \lambda^{2 \alpha} b_{1}(\lambda) d \lambda \int_{r}^{t+\lambda} \frac{d \tau}{\left[\tau^{2}-r^{2}\right]^{\alpha}\left[\lambda^{2}-(t-\tau)^{2}\right]^{1-\alpha}}\right\} .
\end{aligned}
$$

This final result can be cast into the form (2.7a) from which we observe that the PoIsson representation accomplishes everything which VolTERRA's does. Thus 
implicit in the work of Poisson for equation (1.1) is the work of RIEMANn [7], DARBoux [2], and VolTERRA [8]. On the latter score, we note that we have given a verification which is different from DARBoux's. There are also similar results which are associated with (3.2a) and (3.2b) (see Section 2).

\section{The Source-Like Character of the Volterra Representations}

We now examine an interpretation of the functions $f_{i}(\lambda), i=1,3$ and $f_{i}(-\lambda)$, $i=2,4$. We shall see that they are related to various types of "sources" on the axis $r=0$ (with appropriate numerical factors). For the special case $\alpha=1 / 2$ this was observed by LAMB in 1903. We shall examine (3.1 a) and (3.2a) in this connection in terms of the truncated form of the VolterRA representation. Similar results are available for $(3.1 \mathrm{~b})$ and $(3.2 \mathrm{~b})$.

Let us then turn to $\Phi_{1}(t, r)$. We have

$$
\Phi_{1}(t, r)=\int_{0}^{\psi_{0}} f_{1}(t+r \cosh \psi) \sinh ^{2 \alpha-1} \psi d \psi .
$$

If $0<\alpha<1 / 2$, we have

$$
\left|\Phi_{1}(t, r)\right| \leqq \int_{0}^{\psi_{0}}\left|f_{1}(t+r \cosh \psi)\right| \sinh ^{2 \alpha-1} \psi d \psi \leqq M_{1} \int_{0}^{\psi_{0}} \sinh ^{2 \alpha-1} \psi d \psi
$$

where $M_{1}=\max \left|f_{1}(t+r \cosh \psi)\right|, 0 \leqq \psi \leqq \psi_{0}$. Since $f_{1}(t+r \cosh \psi)$ is continuous and

$$
\int_{0}^{\infty} \sinh ^{2 \alpha-1} \psi d \psi
$$

exists for $0<\alpha<1 / 2$, we may let $r \rightarrow 0^{+}$and we have

$$
\Phi_{1}\left(t, 0^{+}\right)=f_{1}(t) \int_{0}^{\infty} \sinh ^{2 \alpha-1} \psi d \psi=\frac{\Gamma(\alpha) \Gamma\left(\frac{1}{2}-\alpha\right)}{2 \sqrt{\pi}} f_{1}(t) .
$$

The numerical factor in (4.1) differs by a factor of $\cos \pi \alpha$ from its Poisson counterpart. It may also be interpreted as a finite part integral for other values of $\alpha$ (see [4], Section 8).

Now we examine $\Phi_{3}(t, r)$. We shall show that for restricted $\alpha, r^{2 \alpha} \partial \Phi_{3} / \partial r$ is proportional to $f_{3}(t)$ when $r \rightarrow 0^{+}$. In order to do so, we calculate $\partial \Phi_{3} / \partial r$ to obtain

$$
\begin{aligned}
\frac{\partial \Phi_{3}}{\partial r}= & (1-2 \alpha) r^{-2 \alpha} \int_{0}^{\psi_{0}} f_{3}(t+r \cosh \psi) \sinh ^{1-2 \alpha} \psi d \psi \\
& +r^{1-2 \alpha} \int_{0}^{\psi_{0}} \frac{\partial f_{3}(t+r \cosh \psi)}{\partial t} \cosh \psi \sinh ^{1-2 \alpha} \psi d \psi \\
& -r^{-2 \alpha} f_{3}(L)\left(\sinh ^{1-2 \alpha} \psi_{0}\right) \frac{(L-t)}{\sqrt{(L-t)^{2}-r^{2}}} .
\end{aligned}
$$


As (4.2) stands, it is not obvious what is the nature of the behavior of $\partial \Phi_{3} / \partial r$ as $r \rightarrow 0^{+}$. In order to cast it into a more useful form, we follow LAMB and write

$$
\begin{aligned}
r^{2 \alpha} \frac{\partial \Phi_{3}}{\partial r}= & (1-2 \alpha) \int_{0}^{\psi_{0}} f_{3}(t+r \cosh \psi) \sinh ^{1-2 \alpha} \psi d \psi \\
& +r \int_{0}^{\psi_{0}} \frac{\partial f_{3}(t+r \cosh \psi)}{\partial t}\left[\sinh \psi+e^{-\psi}\right] \sinh ^{1-2 \alpha} \psi d \psi \\
& -\frac{(L-t) f_{3}(L) \sinh ^{1-2 \alpha} \psi_{0}}{\sqrt{(L-t)^{2}-r^{2}}} .
\end{aligned}
$$

Upon integrating the first term in the second integral by parts and restricting $\alpha$ to the interval $0<\alpha<1 / 2$, we get

$$
\begin{aligned}
r^{2 \alpha} \frac{\partial \Phi_{3}}{\partial r}= & -(1-2 \alpha) \int_{0}^{\psi_{0}}\left[f_{3}(t+r \cosh \psi)\right]\left[e^{-\psi} \sinh ^{-2 \alpha} \psi\right] d \psi \\
& +f_{3}(L)\left[1-\frac{L-t}{\sqrt{(L-t)^{2}-r^{2}}}\right] \sinh ^{1-2 \alpha} \psi_{0} \\
& +r \int_{0}^{\psi_{0}}\left[\frac{\partial f_{3}(t+r \cosh \psi)}{\partial t}\right]\left[e^{-\psi} \sinh ^{1-2 \alpha} \psi\right] d \psi
\end{aligned}
$$

This may be simplified to

$$
\begin{aligned}
r^{2 \alpha} \frac{\partial \Phi_{3}}{\partial r}= & -(1-2 \alpha) \int_{0}^{\psi_{0}}\left[f_{3}(t+r \cosh \psi)\right]\left[e^{-\psi} \sinh ^{-2 \alpha} \psi\right] d \psi \\
& +r \int_{0}^{\psi_{0}} \frac{\partial f_{3}(t+r \cosh \psi)}{\partial t}\left[e^{-\psi} \sinh ^{1-2 \alpha} \psi\right] d \psi \\
& -\frac{r^{1+2 \alpha} f_{3}(L)}{\left[\sqrt{\left.\left.(L-t)^{2}-r^{2}+L-t\right][L-t)^{2}-r^{2}\right]^{\alpha}}\right.}
\end{aligned}
$$

Now since $f_{3}(\lambda) \in C^{2}, \lambda \geqq 0$, it is permissible to let $r \rightarrow 0^{+}$and we are left with

$$
\operatorname{Lim}_{r \rightarrow 0^{+}} r^{2 \alpha} \frac{\partial \Phi_{3}}{\partial r}=-(1-2 \alpha) f_{3}(t) \int_{0}^{\infty} e^{-\psi} \sinh ^{-2 \alpha} \psi d \psi=-\frac{\Gamma(1-\alpha) \Gamma\left(\frac{1}{2}+\alpha\right)}{\sqrt{\pi}} f_{3}(t) .
$$

Let us note that we cannot treat the case $\alpha=1 / 2$ directly from (4.3). In this case we return to (4.2) and observe that the first integral disappears and then we are in a position to apply LAMB's device. In this case we have

$$
\operatorname{Lim}_{r \rightarrow 0^{+}} r \frac{\partial \Phi_{3}}{\partial r}=-f_{3}(t)
$$

This indicates the presence of a conventional two-dimensional "logarithmic source" of strength $f_{3}(t)$ on the $t$-axis. But this is the form of the source strength in the PoIsson representation (1.2a) when $\alpha=1 / 2$ where it indeed displays the logarithmic source explicitly.

The research of Albert HeINS was sponsored by the Air Force Office of Scientific Research, Office of Aerospace Research, United States Air Force, under AFOSR Grant No. 68-1512. 


\section{References}

1. Copson, E. T., On the Riemann-Green function. Arch. Rational Mech. Anal. 1, 324-348 (1958).

2. Darboux, G., Théorie Générale des Surfaces, Vol. 2. Paris: Gauthier-Villars 1889.

3. Friedlander, F. G., Sound Pulses. Cambridge, England: Cambridge University Press 1958.

4. Friedlander, F. G., \& A. E. Heins, On a singular boundary value problem for the EulerDarboux equation. Journal of Differential Equations, forthcoming.

5. LAMB, H., On wave propagation in two dimensions. Proceedings of the London Mathematical Society 35, 141-161 (1903).

6. Porsson, S. D., Sur l'integration des équations linéares aux différences partielle. Journal de l'École Royale Polytechnique XII, Cahier XIX, 215-248 (1823).

7. Riemann, B., Über die Fortpflanzung ebener Luftwellen von Endlicher Schwingungsweite. Abhandlungen der Königlichen Gesellschaft der Wissenschaften zu Göttingen 8 (1860). Reprinted in the Collected Works of Bernard Riemann 156-195, New York: Dover Press 1953.

8. VolterRa, V., Sulle vibrazioni luminose nei mezzi istropi. Rendiconti Accademia Nazionale dei Lincei 1, 161- 170 (1892).

9. Weinstern, A., Singular Partial Differential Equations and Their Applications: Fluid Dynamics and Applied Mathematics. (Proceedings of a Symposium held at the University of Maryland, 1961) 22-49. New York: Gordon and Breach 1962.

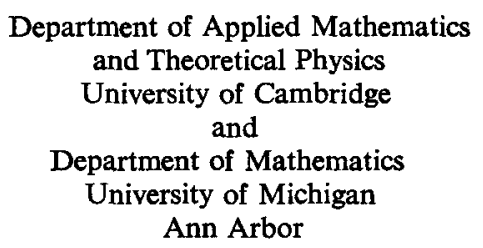

(Received November 5, 1968) 